\title{
Comprehensive Numerical Modeling of Vertical-Cavity Surface-Emitting Lasers
}

\author{
G. Ronald Hadley, Senior Member, IEEE, K. L. Lear, Member, IEEE, \\ M. E. Warren, K. D. Choquette, J. W. Scott, and S. W. Corzine
}

\begin{abstract}
We present a comprehensive numerical model for vertical-cavity surface-emitting lasers that includes all major processes affecting $\mathrm{cw}$ operation of axisymmetric devices. In particular, our model includes a description of the 2-D transport of electrons and holes through the cladding layers to the quantum well(s), diffusion and recombination of these carriers within the wells, the 2-D transport of heat throughout the device, and a multilateral-mode effective index optical model. The optical gain acquired by photons traversing the quantum wells is computed including the effects of strained band structure and quantum confinement. We employ our model to predict the behavior of higher-order lateral modes in proton-implanted devices and to provide an understanding of index-guiding in devices fabricated using selective oxidation.
\end{abstract}

\section{INTRODUCTION}

$\mathbf{V}$ ERTICAL-CAVITY surface-emitting lasers (VCSEL's) are presently the subject of intense research due to their promise as compact, efficient laser sources for a number of important applications. Interest in these devices has been spurred by recent advances in output power [1], efficiency [2], [3], and the extension of operating wavelengths into the visible [4], [5]. These advances, coupled with inherent advantages such as astigmatic output and epitaxially-grown cavities, are placing VCSEL's in a potentially competitive position relative to edge-emitting lasers for the first time. However, further optimization of VCSEL designs will require more elaborate models that are capable of including the many interdependent processes, such as the self-consistent transport of charge carriers, heat and photons, occurring within the device structure.

In this paper, we present such a model for the purpose of understanding the complex physical processes governing cw VCSEL operation, with the ultimate goal of producing a numerical simulation tool capable of designing more efficient and useful devices. In particular, our model includes the following physical processes modeled in 2-D axisymmetric geometry: 1) the ohmic transport of carriers through the cladding layers to a quantum well(s), including anisotropic

Manuscript received March 27, 1995; revised September 1, 1995. This work was performed in part at Sandia National Laboratories and supported by the U.S. Department of Energy under Contract DE-AC04-94AL85000.

G. R. Hadley, K. L. Lear, M. E. Warren, and K. D. Choquette are with Sandia National Laboratories, Albuquerque, NM 87185-5800 USA.

J. W. Scott was with Optical Concepts, Inc., Lompoc, CA 93436 USA. He is now with VIXEL Corporation, Broomfield, CO 80021 USA.

S. W. Corzine was with the University of California, Santa Barbara, CA 93106 USA. He is now with Hewlett Packard Company, Palo Alto, CA 94304 USA.

Publisher Item Identifier S 0018-9197(96)02554-7. conductivities and carrier leakage effects, 2) the transport of heat toward a heat sink, including source terms from ohmic dissipation, nonradiative recombination and reabsorbed laser radiation, 3) the diffusion of carriers inside the quantum well(s) and their recombination via spontaneous emission, stimulated emission, and nonradiative processes (both through traps and Auger transitions), 4) the cw behavior of five different optical cavity modes, delineated by their azimuthal dependence, and 5) the interaction of each of these modes with carriers in the quantum well through a comprehensive gain model that includes effects arising from the strained band structure usually present in the quantum wells. We further validate our model through comparison of predicted performance with measured results for two different types of VCSEL's: 1) gain-guided devices fabricated by ion-implantation and 2) index-guided devices fabricated using selective oxidation.

Most previous VCSEL models [6]-[9] have concentrated on overall device behavior such as threshold and quantum efficiency using simplified treatments of current and heat flow. Such models typically neglect lateral dependencies, due to the obvious difficulty of calculating the 2-D transport, and utilize empirical methods to treat only a few aspects of device behavior. More recently, new models have been reported that attempt a more comprehensive approach. Piprek et al. [10] perform elaborate 2-D carrier and heat transport calculations, but neglect all lateral waveguiding effects. Zhang and Petermann [11] employ a more complete radially-dependent optical model for a single lateral mode, but restrict their carrier and heat transport analysis with the use of simple analytic formulas that are invalid above threshold. Shimizu et al. [12] utilize a two-lateral-mode beam propagation optical model and include gain saturation, but do not treat carrier and heat transport through the cladding layers. Michalzik and Ebeling [13] include 2-D current and heat flow and provide an insightful look at the effects of thermal lensing, but do not include mode competition arising from gain saturation. Thode et al. [14] have presented the most comprehensive model to date, including fully time-dependent 3-D optical, carrier and heat transport models. This very ambitious model is aimed primarily at transient device behavior, and requires extensive mainframe computer resources to implement. The present model concentrates on the phenomena most influential in $\mathrm{cw}$ operation, and includes the major physical processes in a self consistent manner, while requiring modest computer resources (The results reported here were obtained on an IBM RS/6000 model 590 workstation). 
In the following sections, we first derive the relevant governing equations for each of the three component models and describe the physical assumptions employed in their use. Next, we discuss the numerical procedures utilized for the solution of these equations. Finally, the overall model is used to predict device behavior for two different device geometries, and the resulting predictions are compared with actual measured performance. The results of these comparisons are then discussed not only as they relate to model verification, but also in terms of the new insights they provide into VCSEL operation and subsequent design issues.

\section{MATHEMATICAL MODEL}

Transport processes occurring in the VCSEL to be simulated are modeled using the rectangular domain shown in Fig. 1(a). Carriers flow from arbitrarily-tailored contacts through the substrate and cladding layers to the active region, usually composed of one or more quantum wells. Carriers residing in the wells may then diffuse laterally and undergo various recombination processes, while providing gain to the various optical cavity modes. Heat is generated within the device, both due to ohmic heating of carriers during transport and also due to nonradiative processes occurring in the quantum well, as well as reabsorbed radiation. This heat then flows through the device toward a heat sink located either below the substrate or adjacent to the p-cladding layers as shown. The transport of these quantities is computed in the large $(\sim 100 \mu \mathrm{m} \times 100$ $\mu \mathrm{m}$ ) domain shown in Fig. 1(a) using a nonuniform mesh. This domain is kept large so as to adequately treat the spreading of both current and heat as it moves toward the bottom of the substrate.

Optical actiyity, on the other hand, occurs on a more limited spatial scale that is confined to the dashed region shown in Fig. 1(a). The optical field (Fig. 1(b)) is confined vertically by the mirror stacks and laterally by either gain or index guiding. The resulting field is computed on a smaller domain using a separate (and much finer) optical mesh that may be located arbitrarily with respect to the transport mesh. Solution of the optical fields is obtained using an effective index method (as shown schematically in the figure for the case of an etched air-post device) and described in detail below.

The various processes described above are interdependent to varying degrees, thus requiring a highly self-consistent model if accurate predictions are to be obtained. Many of these interdependencies are included in the present model, as depicted in the influence chart shown in Fig. 2. Perhaps the strongest of these is gain saturation, which links carrier transport in the quantum well (the quantum well diffusion model) with the optical model. In addition, the optical model and the heat transport model are linked tbrough thermal lensing and reabsorbed laser radiation. One could also add the effects of band filling on the carrier transport through the cladding layers, but this effect has not been included at present.

\section{A. Heat and Carrier Transport}

The steady-state transport of charge carriers and heat throughout the device is assumed to be linear in nature and

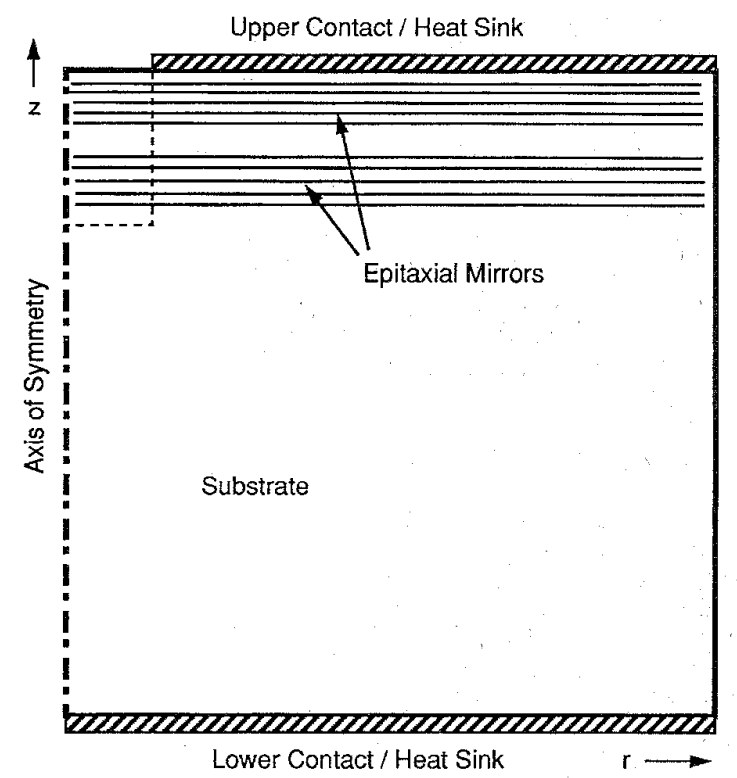

(a)

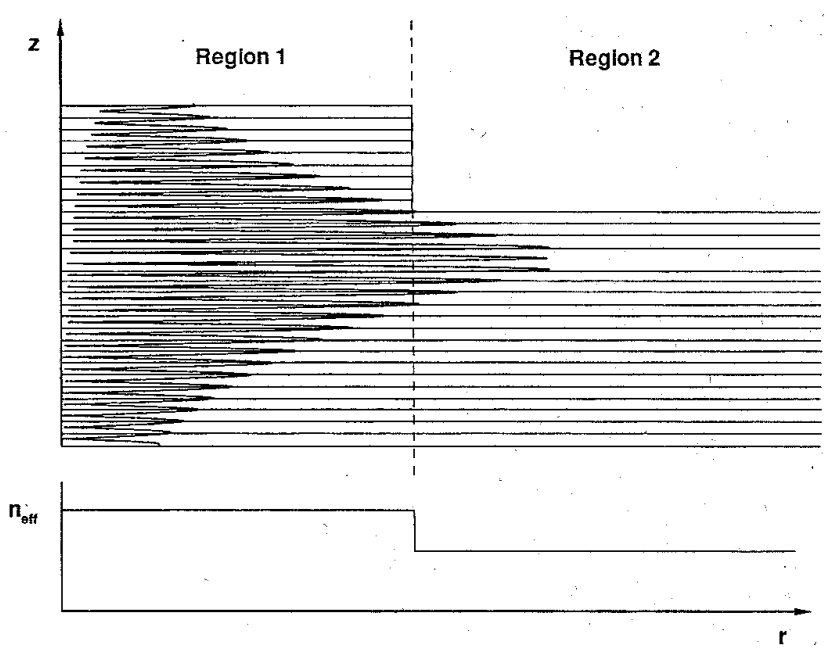

(b)

Fig. 1. Schematic diagram for a generic device simulation showing the computational domain for the transport of heat and carriers (a) and the effective index optical model (b). The optical domain occupies approximately the dashed region in (a).

thus satisfy the general Poisson Equation

$$
\nabla \cdot \bar{\kappa} \nabla \psi=S
$$

where the tensor $\bar{\kappa}$ describes the spatially variable transport properties of the materials, $\psi$ is the transported field quantity, and $S$ is the source. For the heat equation, $S$ is the heat source and $\bar{\kappa}$ the thermal conductivity tensor, assumed to be independent of the temperature $\psi$. For the carrier transport equation, $S$ is zero and $\bar{\kappa}$ is the electrical conductivity tensor, that once again is assumed to be independent of the potential $\psi$. The transport of carriers is also known to depend upon a variety of nonlinear effects, such as thermionic emission, in the vicinity of material interfaces. We will not attempt a 


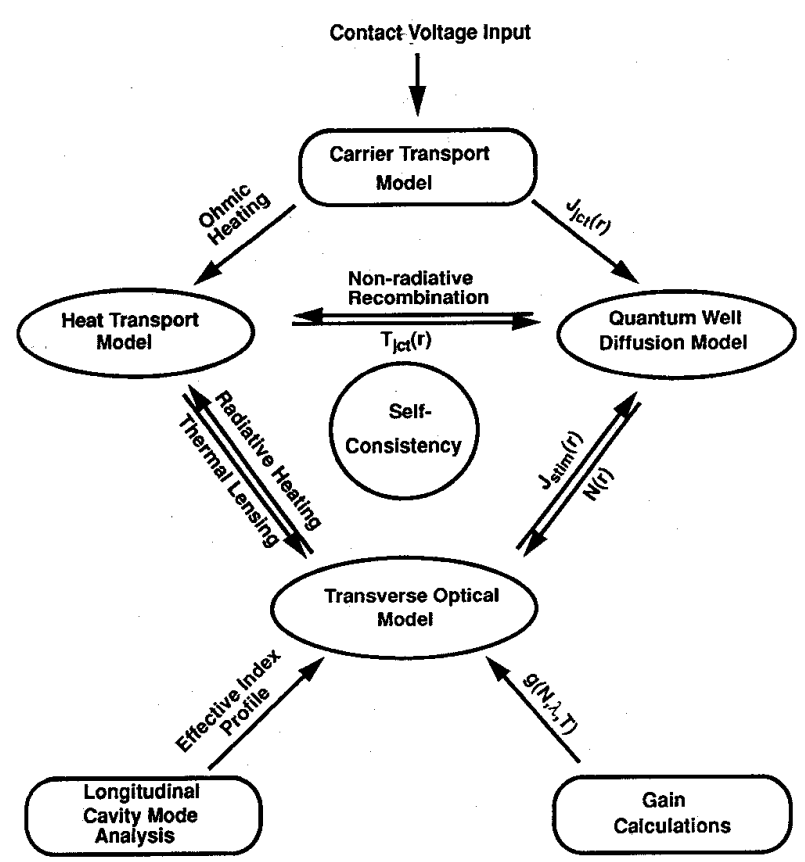

Fig. 2. Flow of information between the various submodels illustrates the complex interdependencies and the need for self-consistent solutions.

detailed treatment of these effects, but rather model transport in the directions normal to and parallel to the interfaces of the DBR mirrors using (1) together with anisotropic average electrical conductivities.

The appropriate source term $S$ for the heat equation describes several different heat generation mechanisms, including ohmic heating by carriers, dissipative recombination processes in the quantum wells, and the reabsorption of both laser and spontaneously-emitted radiation. We include all but the last effect in our treatment, due to the difficulty of calculating its spatial dependence, together with the knowledge that it is relatively diffuse in comparison with the other more concentrated sources [15].

We solve a finite-differenced form of (1) inside a rectangular region of the $r-z$ plane using an iterative procedure that allows mixed boundary conditions to facilitate the simulation of more general heat sink and contact geometries. Internal transport barriers such as implanted regions are modeled by locally decreasing the value of the transport tensor. The finite difference equations we employ are conservative in form and derived starting from an integrated form of (1). Details of both the derivation of these equations as well as the technique employed in their solution is described in detail in the Appendix.

Obtaining the solution of the above general carrier transport equation results in a knowledge of the current density $J(r)$ impinging upon the active region. Carriers thus arriving at the active region that do not leak through the confinement barriers are assumed to diffuse radially within the quantum wells and recombine in a manner described by the $1-\mathrm{D}$ nonlinear
TABLE I

TyPiCAl VAlues Used for Parameters Appearing IN (2)

\begin{tabular}{|l|l|l|}
\hline \multicolumn{1}{|c|}{ Symbol } & \multicolumn{1}{c|}{ Meaning } & \multicolumn{1}{c|}{ Value } \\
\hline \hline$D$ & $\begin{array}{l}\text { Diffusion } \\
\text { Coefficient }\end{array}$ & $10.0 \mathrm{~cm}^{2} / \mathrm{sec}$ \\
\hline$\tau_{n r}$ & $\begin{array}{l}\text { Non-radiative } \\
\text { Recombination } \\
\text { Time }\end{array}$ & $5 \mathrm{nsec}$ \\
\hline$B$ & $\begin{array}{l}\text { Spontaneous } \\
\text { Emission } \\
\text { Coefficient }\end{array}$ & $\begin{array}{l}1.0 \times 10^{-10} \\
\mathrm{~cm}^{3} / \mathrm{sec}\end{array}$ \\
\hline$C$ & $\begin{array}{l}\text { Auger } \\
\text { Coefficient }\end{array}$ & $\begin{array}{l}3.5 \times 10^{-30} \\
\mathrm{~cm}^{6} / \mathrm{sec}\end{array}$ \\
\hline
\end{tabular}

ambipolar diffusion equation

$$
\begin{aligned}
\frac{D}{r} \frac{d}{d r}\left(r \frac{d N}{d r}\right)= & -\frac{J_{\mathrm{eff}}(r)}{q d}+\frac{N(r)}{\tau_{n r}}+B N^{2}+C N^{3} \\
& +\frac{g_{\mathrm{eff}}(r)}{h \nu d} \sum_{m=1}^{M}\left|E_{m}(r)\right|^{2} .
\end{aligned}
$$

where $N$ is the density of electrons and holes in the quantum well, $D$ is the (constant) diffusion coefficient, $q$ the magnitude of the electronic charge, $d$ the effective quantum well width (the total width for multiple quantum wells), $\tau_{n r}$ the nonradiative recombination time, $B$ the coefficient for spontaneous emission, $C$ the Auger coefficient, $h \nu$ the photon energy, and $g_{\text {eff }}$ the effective gain coefficient. $J_{\text {eff }}$ represents the remaining current density after leakage current is subtracted off. The leakage current density is estimated through the approximate relation [7]

$$
J_{\text {leak }}=J_{0} e^{\frac{\left(E_{f}-E_{b}\right)}{k T}} .
$$

In (3), $E_{b}$ is the effective barrier height, $E_{f}$ the quasi-Fermi level separation, and $J_{0}$ is determined by a fit of threshold versus temperature data for a particular device structure. Representative values for these parameters are reported in [7], although the current leakage model was not employed for the calculations reported here since the devices being modeled were designed to have large values of $E_{b}$. Referring back to (2), the gain coefficient $g$ depends on radius through its dependence upon carrier density, lasing wavelength, and temperature via an elaborate gain model to be described in a later section. The lasing intensity term is summed incoherently over all lateral modes present, since each lases at a different frequency. Typical values employed for the above parameters are listed in Table I.

We produce a finite-differenced form of (2) for the carrier density $N_{i}$ by premultiplication by $r$ and integration over the range $r_{i-(1 / 2)}$ to $r_{i+(1 / 2)}$. This equation is then linearized by expansion about the previous solution at each mesh point. The resulting linear equation is then solved using the Thomas tridiagonal algorithm and the iterative process repeated until convergence is obtained. Due to the strong nonlinearities involved, under-relaxation is used to prevent gain overshoot and undershoot. 


\section{B. Optical Model}

Lasing modes in a vertical cavity laser are characterized by near-paraxial propagation normal to the mirror layers, with polarizations in the plane of the mirrors. Under these conditions, only a single electric field component is appreciable, and we may describe that field adequately with the scalar wave equation

$$
\nabla^{2} F-\frac{\epsilon}{c^{2}} \frac{\partial^{2} F}{\partial t^{2}}=0
$$

where $\epsilon$ is the relative permittivity, and we have assumed the relative permeability to be unity. Since $F$ depends on all three spatial coordinates and time, we cannot easily solve (4) for a complicated structure such as a VCSEL. Instead, we invoke two simplifying assumptions: (1) that the time dependence is nearly harmonic with (complex) frequency $\omega_{0}$, and (2) that the VCSEL structure depends only upon $z$ within each of a number of concentric cylindrical regions. Thus, we write for region $i$,

$$
F(r, z, \phi, t) \approx \varphi_{i}(z) E(r, \phi, t) e^{-i \omega_{0} t} .
$$

Substituting (5) into (4) and writing $\nabla^{2}=\partial^{2} / \partial z^{2}+\nabla_{\perp}^{2}$ results in

$$
\varphi_{i}^{\prime \prime} E+\varphi_{i} \nabla_{\perp}^{2} E+\epsilon k_{0}^{2} \varphi_{i} E+2 \dot{i} \epsilon k_{0} \varphi_{i} \frac{\partial E}{\partial \tau}=0,
$$

where we have introduced the vacuum wavevector $k_{0} \equiv \omega_{0} / c$ and the modified time coordinate $\tau \equiv c t$, and have invoked the slowly-varying envelope approximation by neglecting second derivatives with respect to time when acting on the function $E$. In anticipation of the separability assumption, we next write the relative permittivity as the sum of a structural component $\epsilon_{i}$ and a nonstructural component $\epsilon_{g}$ that is expected to include such effects as radially-dependent gain in the quantum well, and thermal lensing:

$$
\epsilon(r, z) \equiv \epsilon_{i}(z)+\epsilon_{g}(r)
$$

At this point, we introduce the effective index approximation by assuming that (1) the functions $\varphi_{i}$ each satisfy a 1-D eigenvalue equation of the form

$$
\varphi_{i}^{\prime \prime}+k_{0}^{2}\left(1-\xi_{i}\right) \epsilon_{i}(z) \varphi_{i}=0,
$$

where the complex eigenvalue $\xi_{i}$ is related to the effective index, and (2) that the eigenfunctions $\varphi_{i}$ are all approximately identical in each of the problem regions, so that for distinct regions $i$ and $j, \varphi_{i}(z) \approx \varphi_{j}(z) \equiv \varphi(z)$. The latter statement embodies the essence of the effective index method by implying approximate separability, with the dominant effect of structure variations appearing primarily as a variation in the effective index. Consequently, we might expect the model to give inaccurate results when applied to devices with severe structural variations that violate separability. Such effects are seen in the simulations of the index-guided VCSEL's described below.

If we now use (8) to eliminate the $\varphi^{\prime \prime}$ term in (6), we have

$$
k_{0}^{2}\left(\epsilon_{g}(r)+\xi \epsilon_{i}(z)\right) \varphi E+\varphi \nabla_{\perp}^{2} E+2 i k_{0} \epsilon_{i}(z) \varphi \frac{\partial E}{\partial \tau}=0
$$

The $z$-dependence in (9) may be removed by multiplying by $\varphi^{*}$ and integrating over $z$, resulting in

$$
k_{0}^{2}\left(\left\langle\epsilon_{g}\right\rangle+\xi\left\langle\epsilon_{i}\right\rangle\right) E+\nabla_{\perp}^{2} E+2 i k_{0}\left\langle\epsilon_{i}\right\rangle \frac{\partial E}{\partial \tau}=0,
$$

where we have defined

$$
\left\langle\epsilon_{i}\right\rangle \equiv \frac{\int \varphi^{*} \epsilon_{i}(z) \varphi d z}{\int \varphi^{*} \varphi d z}
$$

Equation (10) may be rewritten in the form of a beam propagation equation where the propagation direction is the time-like variable $\tau=c t$. The result is

$$
\frac{\partial E}{\partial \tau}=\frac{i}{2 k_{0}\left\langle\epsilon_{i}\right\rangle}\left(\nabla_{\perp}^{2}+k_{0}^{2} \Delta \epsilon_{\mathrm{eff}}\right) E
$$

where we have defined the variation in effective dielectric constant as $\Delta \epsilon_{\mathrm{eff}} \equiv\left\langle\epsilon_{g}\right\rangle+\xi_{i}\left\langle\epsilon_{i}\right\rangle$ and $\left\langle\epsilon_{g}\right\rangle$ is given by

$$
\left\langle\epsilon_{g}\right\rangle=2 \sqrt{\operatorname{Re}\left(\left\langle\epsilon_{i}\right\rangle\right)} \frac{d n}{d T}\left(\langle T\rangle(r)-T_{0}\right)-\frac{i g n_{a}\langle u\rangle}{k_{0}},
$$

where $d n / d T$ is the derivative of the refractive index with respect to temperature, $g$ and $n_{a}$ are the material gain coefficient and index of refraction of the quantum wells, and $u$ is a function that is unity in the quantum wells and zero otherwise. We have ignored changes in refractive index due to the presence of carriers in (13), because the small value of $\langle u\rangle$ renders those changes small $\left(5 \times 10^{-4}\right)$ compared with those due to thermal lensing $\left(\sim 5 \times 10^{-3}\right)$. Also, we neglect the dependence of the parameter $d n / d T$ upon aluminum composition, interpreting its value as a material average.

Note that despite the familiar form of (12), it is in reality an expression of time variation, so that the model we are espousing is not a true beam propagation model. This distinction is clarified by the observation that in our model there is no necessity of defining an effective mirror penetration depth, as is the case with true beam propagation models [12]. This is of considerable advantage for calculating the losses incurred, for example, by free carrier absorption resulting from mirrors grown using arbitrary doping profiles. The inclusion of such losses is awkward with a beam propagation approach, but appears naturally in our model as a contribution to the imaginary part of the eigenvalue $\xi_{i}$.

Our optical model thus consists of two parts: (1) initial solutions of (8) for each region that result in the eigenvalues $\xi_{i}$, and (2) iterative solutions of (12) for each lateral mode that continue until both the radial dependence and magnitude of $E$ (proportional to the square root of the recirculating power) cease to change. Different lateral modes are defined by requiring cylindrical symmetry for the field $E$. Thus, $E$ must be of the form

$$
E(r, \phi, t) \equiv E_{m}(r, t) e^{ \pm i m \phi} .
$$

Inserting (14) into (12) and writing the Laplacian in cylindrical coordinates results in the following propagation equation for the $m$ th lateral mode:

$$
\frac{\partial E_{m}}{\partial \tau}=\frac{i}{2 k_{0}\left\langle\epsilon_{i}\right\rangle}\left(\frac{1}{r} \frac{\partial}{\partial r} r \frac{\partial}{\partial r}-\frac{m^{2}}{r^{2}}+k_{0}^{2} \Delta \epsilon_{\mathrm{eff}}\right) E_{m}
$$


Equation (15) is discretized on a nonuniform mesh and solved using the Thomas tridiagonal algorithm.

Before continuing, we shall digress temporarily to explore both some useful relations and some important concepts that proceed naturally from (5)-(12). First, we examine more closely the information contained in the field profile $\varphi(z)$ and its eigenvalues $\xi_{i}$. To this end, we first consider a 1-D cold cavity, ignoring any radial dependence for the moment. The field in such a cavity may be described by (12) with the Laplacian operator deleted. The solution (expressed as a time dependence) is thus

$$
E \sim e^{i \omega_{0} \xi t / 2}
$$

and if we include the explicit time dependence assumed in (5), we have

$$
E \sim e^{-i \omega_{0} t(1-(\operatorname{Re}(\xi) / 2))-\left(\omega_{0} \operatorname{Im}(\xi) t / 2\right)} .
$$

Thus, the energy stored in the cavity decays as

$$
U \sim e^{-\omega_{0} \operatorname{Im}(\xi) t},
$$

and the cavity $Q$ is thus $Q=1 / \operatorname{Im}(\xi)$ [17]. Therefore, we see that the real part of the eigenvalue $\xi$ corresponds to a shift in the Fabry-Perot resonance of the cavity relative to the initial guess $\omega_{0}$, and the imaginary part describes the cavity losses. In order to calculate the radiated power, we need to distinguish between radiative losses through the mirrors and nonradiative losses due to absorption in the dielectric layers. This is accomplished by multiplication of (8) (without subscripts) by $\varphi^{*}$, its conjugate by $\varphi$, and subtracting. The result, after an integration by parts, is

$$
\left[\varphi^{*} \frac{d \varphi}{d z}-\varphi \frac{d \varphi^{*}}{d z}\right]_{l}^{u}+2 i k_{0}^{2} \operatorname{Im}[(1-\xi)\langle\epsilon\rangle] \int \varphi^{*} \varphi d z=0
$$

where the subscript and superscript around the brackets denote the upper and lower problem boundaries. Now we assume that each end of the cavity is terminated in a single lossless dielectric, so that in those regions

$$
\begin{aligned}
\varphi_{u} & \sim e^{i k_{0} n_{u} z} \\
\varphi_{l} & \sim e^{-i k_{0} n_{l} z}
\end{aligned}
$$

in which case (19) may be solved for the imaginary part of $\xi$, yielding

$$
\begin{aligned}
\operatorname{Im}(\xi)= & \frac{\operatorname{Im}(\langle\epsilon\rangle)(1-\operatorname{Re}(\xi))}{\operatorname{Re}(\langle\epsilon\rangle)}+\frac{n_{u}|\varphi|_{u}^{2}}{k_{0} \operatorname{Re}(\langle\epsilon\rangle) \int \varphi^{*} \varphi d z} \\
& +\frac{n_{l}|\varphi|_{l}^{2}}{k_{0} \operatorname{Re}(\langle\epsilon\rangle) \int \varphi^{*} \varphi d z}
\end{aligned}
$$

The first term in (21) describes absorption loss, being proportional to a weighted average of the imaginary part of the dielectric constant of each layer. The remaining terms describe radiation losses at the upper and lower problem boundaries, with material indexes $n_{u}$ and $n_{l}$, respectively. Returning to (17), we see that a nonzero value for the real part of the eigenvalue corresponds with a shift in the cavity resonance frequency given by

$$
\frac{\Delta \omega}{\omega_{0}}=-\frac{\Delta \lambda}{\lambda_{0}}=\frac{\operatorname{Re}(\xi)}{2} .
$$

But we previously noted that the change in effective dielectric constant arising from the real part of $\xi$ was

$$
\frac{\Delta \epsilon_{\mathrm{eff}}}{2\langle\epsilon\rangle} \approx \frac{\Delta n_{\mathrm{eff}}}{n_{\mathrm{eff}}}=\frac{\operatorname{Re}(\xi)}{2} .
$$

Combining these results leads to the important connection that a shift of the cavity resonance in one radial region relative to another leads to a change in effective index. The magnitude of this change is given by

$$
\frac{\Delta n_{\mathrm{eff}}}{n_{\mathrm{eff}}}=\frac{\Delta \lambda}{\lambda_{0}}
$$

and can be substantial. For example, a $10 \mathrm{~nm}$ red-shift in cavity resonance for a VCSEL operating at $975 \mathrm{~nm}$ corresponds to an effective index increase of about 0.033 , a large value when compared with the index contrast of $\sim 10^{-3}$ typically required for index guiding in rib waveguides. Thus, our model leads naturally to the surprising conclusion that the relative change in effective index in a certain region of a vertical cavity laser is due entirely to a shift in the Fabry-Perot resonance frequency in that region, with red (blue) shifts leading to increases (decreases) in effective index, respectively. Thus, an alteration in index of the material making up the cavity affects the index by altering the cavity resonance frequency. This statement can be understood heuristically in terms of a simple optical cavity made up of a single material, whose optical path length is $n L$. An increase in cavity length with constant index is seen to be equivalent to a proportionate increase in index with cavity length held constant. Of course, an increase in the effective cavity length red-shifts the Fabry-Perot resonance wavelength proportionately, so the two pictures are consistent. These conclusions are reinforced by the experimental data to be discussed in a later section, and are expected to lead to the design of future VCSEL's with improved modal properties.

Up to this point, all field expressions have been written in such a way as to be independent of normalization. The latter must however be determined consistently with the stimulated emission term in (2) so that the recombination of one electronhole pair produces exactly one photon. This condition is properly met if we define the effective gain coefficient in (2) as

$$
g_{\text {eff }} \equiv \frac{n_{a} g\langle u\rangle}{k_{0} \operatorname{Re}\left(\left\langle\epsilon_{i}\right\rangle\right)}
$$

and the radiated output power density by

$$
\left.P_{\text {out }} \equiv \operatorname{Im}\left(\xi_{i}\right)\right|_{\mathrm{rad}}|E|^{2} \text {. }
$$

With the above choice of normalization, $|E|^{2}$ is proportional to the recirculating power density, having units of $W / \mathrm{cm}^{2}$. We note in passing that the expression for the effective gain coefficient in (25) is a weighted average and therefore automatically accounts for resonant-periodic-gain effects, yielding zero gain if the quantum well is placed exactly at a field node. 


\section{Gain Model}

The material gain coefficient $g(N, \lambda, T)$ appearing in (13) is determined from detailed band structure calculations for the 8nm-wide compressively-strained $\operatorname{In}_{0.2} \mathrm{Ga}_{0.8}$ As quantum wells. The details of these calculations have been reported elsewhere [18], [19], and will be briefly summarized here for the sake of completeness. Due to the level of sophistication of these calculations, they are performed only once for a given quantum well structure with the results being stored in lookup tables or fitted by analytic functions. These tables or functions are then employed in the VCSEL simulations.

These calculations begin with a determination of the subband structure of the strained quantum well for both conduction and valence bands. The quantized subband edge energy levels in both bands are determined by solving Schroedinger's Equation with a finite barrier potential appropriate for the $\mathrm{In}_{0.2} \mathrm{Ga}_{0.8} \mathrm{As} / \mathrm{GaAs}$ system $\left(E_{G}(\mathrm{GaAs}: \mathrm{C}-\mathrm{HH}, \mathrm{LH})=1.424 \mathrm{eV}\right.$ and $E_{G}\left(\mathrm{In}_{0.2} \mathrm{Ga}_{0.8} \mathrm{As}\right.$ :strained C-HH) $=1.215 \mathrm{eV}$ with $60 \%$ of the bandgap discontinuity assumed to appear in the conduction band). In the valence band, a different potential well is seen by heavy and light holes due to strain [18]. The three potential well depths used are: $V_{C}=125 \mathrm{meV}, V_{H H}=83.6 \mathrm{meV}$, and $V_{L H}=3.75 \mathrm{meV}$ (due to the strain-induced splitting of the heavy and light hole bands, the light holes are nearly unconfined). The resulting quantized energies in the quantum well are: $E_{C n}=37.4,122, E_{H H n}=9.61,37.3,76.2$, and $E_{L H n}=83.1$, all in $\mathrm{meV}$.

The next step involves determining the subbands associated with each quantized energy level. In the conduction band, each subband is assumed to be parabolic in our model, with an inplane effective mass that is a weighted average of the band edge conduction band effective masses in and out of the well $\left(0.059 m_{0}\right.$ and $0.067 m_{0}$, respectively). In the valence band, a realistic model must account for the complex interactions between the heavy and light hole subbands that lead to subband warping. To include these valence band-mixing effects, the Luttinger-Kohn (L-K) Hamiltonian [20] is employed. In our model, we use the $4 \times 4$ version of the L-K Hamiltonian that takes into account the coupling between the heavy and light hole valence bands, but neglects the coupling to the split-off band (aside from strain shifts in the bandgaps that do include split-off band coupling).

A unity transformation applied to the $4 \times 4$ L-K Hamiltonian transforms it into two $2 \times 2$ decoupled Hamiltonians, making the analysis much simpler, as first suggested by Broido and Sham [21] (and outlined in more detail by Ahn and Chuang [22]). After adding the' strained heavy hole and light hole finite barrier potential well profiles to the L-K Hamiltonian (in a manner equivalent to that described in [22]), we can solve for the quantum well envelope functions. The corresponding energy eigenvalues as a function of in-plane $k$ vector are then found successively, producing the entire valence subband structure.

Once the subband structure is determined, we can concentrate on the more relevant topic of calculating the gain function. To predict the gain/absorption characteristics of quantum well structures in general, we need to know (1) the density of states in both the conduction and valence bands, (2) the interband transition matrix elements, and (3) the quasi-Fermi levels in both bands. With the subband structure results as described above, we can easily calculate the density of states. A knowledge of the envelope functions combined with the strength of the bulk momentum matrix element (for $\mathrm{In}_{0.2} \mathrm{Ga}_{0.8}$ As we assumed $2|M|^{2} / m_{0}=27.48 \mathrm{eV}$ ) allows a determination of the interband transition matrix element as a function of in-plane $k$ vector for every subband transition pair [18].

This leaves only the task of determining the quasi-Fermi levels in each band. In our model, we treat the conduction band quasi-Fermi level as the independent variable of the calculation and relate the valence band quasi-Fermi level to it by invoking charge neutrality in the quantum well region (including "bulk" states at energies beyond the potential barrier heights). With these defined, we can uniquely determine the carrier density within each band and the gain/absorption spectrum of the quantum well. Spectral broadening of the transitions is taken into account by convolving the resulting gain spectrum with an energy-dependent lineshape function [23] (it is similar to a Lorentzian with an intraband scattering time of $0.1 \mathrm{ps}$, with the exception that the function used here is reduced by more than an order of magnitude "in the wings" as compared to the Lorentzian). In this way, the carrier density and broadened gain spectrum can be found as a function of increasing quasi-Fermi level separation.

The resulting gain model has been compared with experiment by integrating the total emission rate over all possible transitions as calculated above, and comparing calculated gain versus radiative current density with measured values for edge-emitting lasers employing both single and double $\mathrm{In}_{0.2} \mathrm{Ga}_{0.8} \mathrm{As} / \mathrm{GaAs} 8$-nm-wide quantum wells. The resulting comparisons, shown in Fig. 3, are excellent [24], particularly in view of the fact that the calculations employed no fitting parameters.

\section{COMPARISON WITH EXPERIMENT}

We illustrate the use and demonstrate the validity of the model described in this paper by comparing model predictions against measured device performance for two different types of vertical cavity lasers. The first is a gain-guided device with no built-in index structure whose lateral mode shape is determined by the shape of the gain profile. The second is an index-guided laser whose index step results from the insertion of a low-index layer using selective oxidation. This device offers a challenging test of the effective index model described above. Problem-dependent parameters used to simulate these two devices are listed in Table $\Pi$.

\section{A. Gain-Guided VCSEL}

The devices used for this comparison were processed from molecular-beam-epitaxially-grown wafers of AlGaAs mirrors on n-type GaAs substrates with triple InGaAs quantum wells, as described in detail elsewhere [2]. Gain-guided VCSEL's with circular symmetry and diameters ranging from $10-35 \mu \mathrm{m}$ were then defined by proton bombardment, resulting in device 


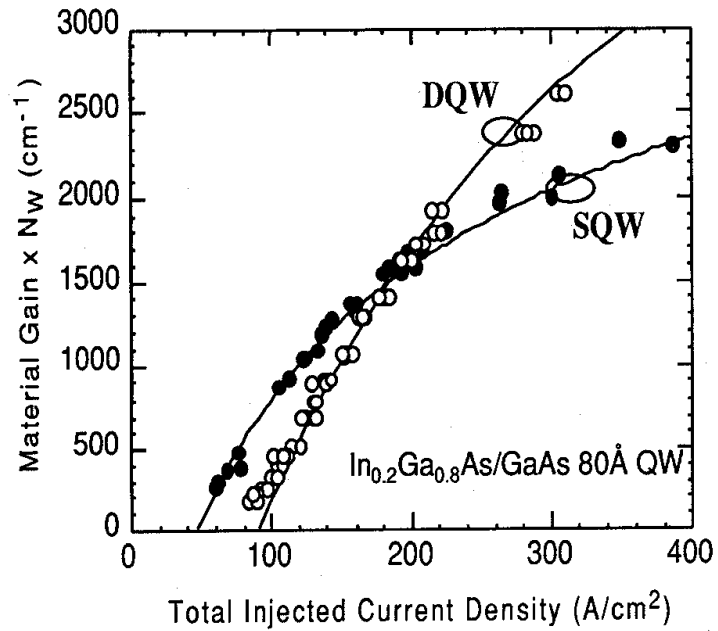

Fig. 3. A comparison between theoretical gain and measured gain (taken from different length devices) as a function of current density in single and double $\mathrm{In}_{0.2} \mathrm{Ga}_{0.8} \mathrm{As} / \mathrm{GaAs} 8 \mathrm{~nm}$ quantum well active region edge-emitting lasers (after [24]). The theoretical plot includes current contributions from Auger and barrier recombination.

TABLE II

Parmaeters Employed in the Device Simulations Discussed in the Text. Transport Quantities Represent Fits, and Deviate in Some Cases from Typical Published Values

\begin{tabular}{|c|c|c|}
\hline Property & Gain-Guided Simulation & Index-Guided Simulation \\
\hline $\begin{array}{l}\text { Optical Cavity: } \\
\text { Lower Mirror Periods } \\
\text { Upper Mirror Periods } \\
\text { Cavity Length }\end{array}$ & $\begin{array}{l}30 \\
22 \\
1 \lambda\end{array}$ & $\begin{array}{l}38 \\
18 \\
1 \lambda\end{array}$ \\
\hline $\begin{array}{l}\text { Central Region: } \\
\text { Resonant Wavelength } \\
\text { Quantum Well Overlap } \\
\text { Round Trip Loss }\end{array}$ & $\begin{array}{l}975.7 \mathrm{~nm} \\
0.0269 \\
1.58 \%\end{array}$ & $\begin{array}{l}966.8 \mathrm{~nm} \\
0.0337 \\
0.64 \%\end{array}$ \\
\hline $\begin{array}{l}\text { Implanted/Oxidized Region: } \\
\text { Resonant Wavelength } \\
\text { Quantum Well Overlap } \\
\text { Round Thip Loss }\end{array}$ & $\begin{array}{l}975.7 \mathrm{~nm} \\
0.0269 \\
4.41 \%\end{array}$ & $\begin{array}{l}948.4 \mathrm{~nm} \\
0.0365 \\
0.4 \%\end{array}$ \\
\hline $\begin{array}{l}\text { Electrical Transport: } \\
\text { P-Type Mirror Conduct. } \\
\text { Implanted/Oxidized Region: } \\
\text { thickness } \\
\text { conductivity } \\
\text { N-Type Mirror Conduct. } \\
\text { Substrate Conductivity }\end{array}$ & $\begin{array}{l}\text { (Conductivities in Mho } / \mathrm{cm} \text { ) } \\
\sigma_{r}=10 \quad \sigma_{z}=5 \\
1.5 \mu \mathrm{m} \\
\sigma_{r}=10^{-4} \quad \sigma_{z}=10^{-4} \\
\sigma_{r}=100 \quad \sigma_{z}=1.5 \\
\sigma_{r}=500 \quad \sigma_{z}=500\end{array}$ & $\begin{array}{l}\sigma_{r}=7.5 \quad \sigma_{z}=4 \\
0.1 \mu \mathrm{m} \\
\sigma_{r}=2 \times 10^{-6} \quad \sigma_{z}=2 \times 10^{-6} \\
\sigma_{r}=75 \quad \sigma_{z}=1.2 \\
\sigma_{r}=500 \quad \sigma_{z}=500\end{array}$ \\
\hline $\begin{array}{l}\text { Thermal Transport: } \\
\text { Mirror Conductivities } \\
\text { Substrate Conductivity } \\
\text { Temp dependence of Cond. } \\
\text { Contact metal Conductivity }\end{array}$ & $\begin{array}{l}\text { (Conductivities in W/cmK) } \\
k_{r}=0.08 \quad k_{z}=0.03 \\
k_{r}=0.39 \quad k_{z}=0.39 \\
k_{\infty} \propto T^{-1.2} \\
k_{r}=1.0 \quad k_{z}=1.0\end{array}$ & $\begin{array}{l}k_{r}=0.125 \quad k_{z}=0.075 \\
k_{r}=0.39 \quad k_{z}=0.39 \\
k_{r}=1.0 \quad k_{z}=1.0\end{array}$ \\
\hline
\end{tabular}

geometries shown schematically in Fig. 4. We modeled a simplified version of the devices in which the triple quantum wells were assumed to have equal carrier density profiles [6]. The electrical resistivities were modified to reproduce the measured device resistance for several different device diameters. Likewise, the thermal conductivities were modified [25] to reproduce the measured device temperature rise. The latter quantity was determined by first observing the Fabry-Perot resonance shift (at zero device current) as the temperature of the mounting stage was varied. This procedure provided not only the relationship between device temperature and emission wavelength, but also the material parameter $d n / d T$, which

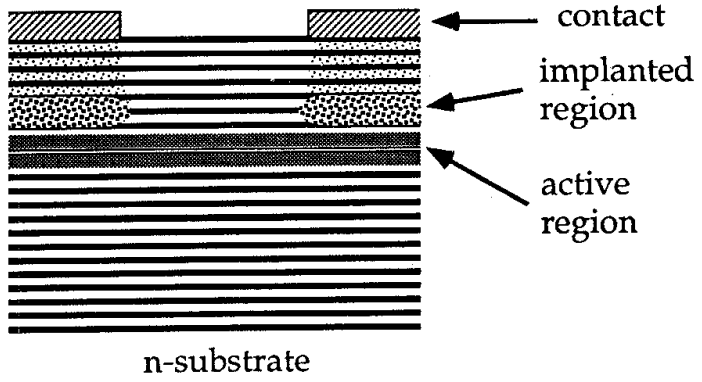

Fig. 4. Schematic diagram of the structure of an implanted gain-guided device. The implanted region is assumed to have negligible effect on the optical properties, but have very low electrical conductivity.

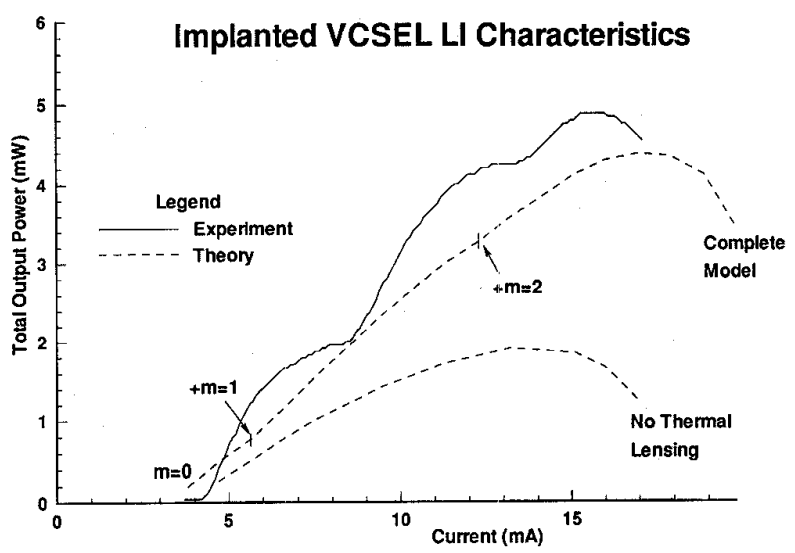

Fig. 5. Comparison between model results and data for the implanted gain-guided device. The model predicts only the fundamental mode at low power, with higher-order lateral modes coming above threshold at the points shown. The onset of higher-order modes in the data is shown by the kinks in the curve. The bottom theoretical curve computed with thermal lensing turned off shows lower quantum efficiency and only the fundamental mode.

was found to have a value of $2.3 \times 10^{-4}$. The device was then simulated using the model described in the previous sections and the measured value of $d n / d T$. Optical losses were increased in the implanted region to model the effects of the ion implantation. The resulting predicted LI curves for the $15-\mu \mathrm{m}$-diameter device are shown in Fig. 5 along with the measured results. The simulation clearly predicts the onset of two higher-order lateral modes at the currents shown. These two modes were also observed experimentally, appearing at currents corresponding to the kinks in the curve marked "Experiment" in Fig. 5 (and confirmed by near-field and spectral measurements). In addition, the calculated results accurately predict the measured threshold current and differential quantum efficiency, and approximately predict the observed thermal rollover. Further calculations performed with thermal lensing effects removed show a decreased differential quantum efficiency and only a single lateral mode (Fig. 5). The thermal lens is clearly seen in the isotherms corresponding to a current of $14.5 \mathrm{~mA}$, shown in Fig. 6.

These results demonstrate clearly the crucial role played by thermal lensing in determining the $\mathrm{cw}$ modal behavior of implanted devices. The thermal lens acts to pull the higherorder modes into the gain region, where they compete more effectively with the fundamental mode. It also leads to tighter 


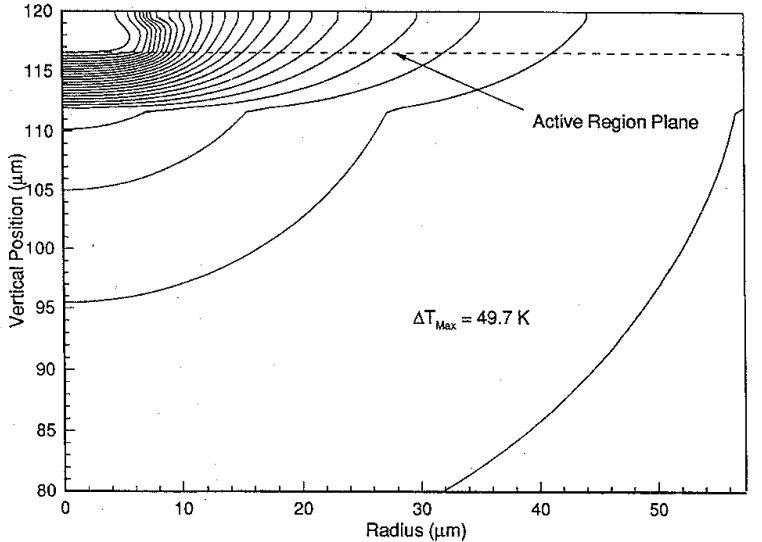

Fig. 6. Isotherms for the simulation of the gain-guided implanted device corresponding to a current of $14.5 \mathrm{~mA}$. The presence of a thermal lens is clearly seen as a decrease in temperature along the plane of the active region. Discontinuities in the isotherms reflect decreased values of thermal conductivity in the mirror regions [25] needed to reproduce measured device temperature rises.

confinement of the fundamental mode, with the resulting higher efficiency.

\section{B. Index-Guided VCSEL}

Devices used for this paper were processed from wafers grown using MOVPE of a design similar to that previously used to make implanted devices. The wafers differed only in the use of fewer upper mirror pairs, and the modification of the aluminum content of one mirror layer adjacent to the cavity from 96 to $98 \%$. These wafers were processed by first etching large rectangular mesas followed by oxidation as described elsewhere [3]. The slight difference in $\mathrm{Al}$ content of the mirror layers resulted in the preferential oxidation of the single $98 \%$ layer, and the consequent formation of a current aperture with a rectangular shape of varying dimensions as shown in Fig. 7. The lower index of refraction of the oxide layer also results in the reduction of the effective index in the oxidized region of the VCSEL as computed using (8). The magnitude of the resulting index step has been computed to be approximately 0.066 , a large enough value to cause highly effective optical confinement. This is also in good agreement with the value of 0.062 inferred from a measurement of lateral mode spacing below threshold [26].

We chose two devices for comparison whose apertures were squares with sides of approximately 3 and $7 \mu \mathrm{m}$, and simulated their performance using the model described above as circular devices with radii of 1.5 and $3.5 \mu \mathrm{m}$, respectively. The two simulations employed identical properties, with only the oxidation diameter being varied. The resulting measured and calculated LI curves are shown in Figs. 8 and 9. As can be seen, the $7 \mu \mathrm{m}$-diameter simulation matches the data very closely, with the predicted threshold $(240 \mathrm{~mA})$ slightly under the measured value of $350 \mathrm{~mA}$, and the maximum wallplug efficiency $(46 \%)$ just under the world-record measured efficiency of $50 \%$ [3]. The observed single-lateral-mode operation to $1.5 \mathrm{~mW}$ output power was not, however, reproduced in the simulation, which predicted higher-order lateral modes just

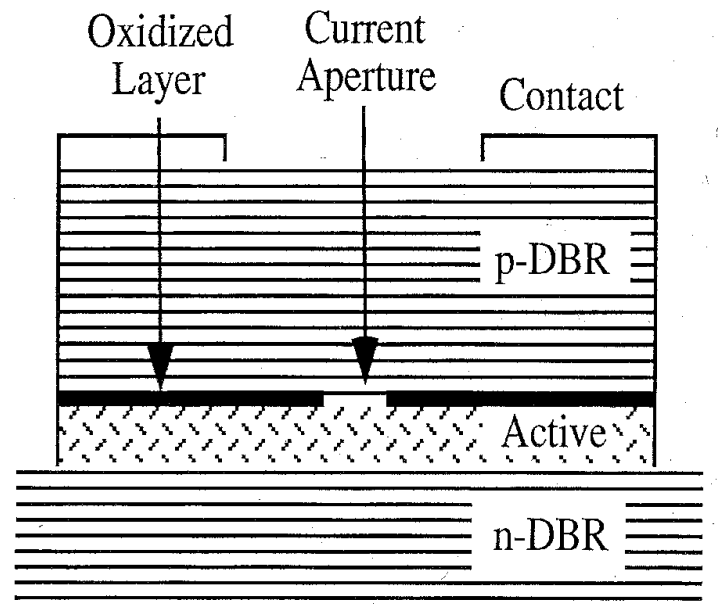

Fig. 7. Schematic diagrami of the structure of an index-guided device fabricated using selective oxidation of a single quarter-wave mirror layer. The oxide functions both as a means of current confinement and as an index guide.

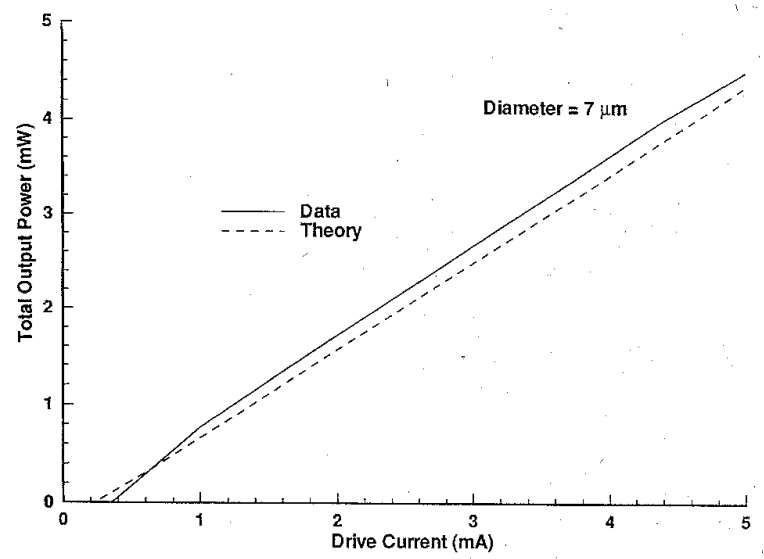

Fig. 8. Measured and predicted LI curves for a 7- $\mu \mathrm{m}$-diameter index-guided device of the type shown in Fig. 7. This device lases at a maximum wallplug efficiency of ( $46 \%) 52 \%$ at $2 \mathrm{~mA}$ drive current as determined by (calculations) measurement.

above threshold. For the $3 \mu \mathrm{m}$-diameter device (see Fig. 9), the simulations predict lower threshold current and higher quantum efficiency than were measured in the laboratory. Also, they predict the lasing of two lateral modes as opposed to the observed single mode operation. It thus appears that the oxide layer is introducing an extra mode-dependent loss into the cavity that is not being accounted for by the effective index formalism. We believe that this loss results because the lowindex oxide layer represents a lumped rather than a distributed index change that causes both scattering and confinement. The scattering would be expected to be greater for higher-order lateral modes that more strongly overlap the oxidized region, and is not predicted by the effective index formalism since the latter describes an average (or distributed) change in index. Inclusion of this loss mechanism would bring the $3-\mu \mathrm{m}$ LI results more closely into agreement with the observations, and also discriminate against the operation of higher-order lateral modes. Thus, we conclude that more accurate modeling of such strongly index-guided devices will require a more sophisticated optical model. 


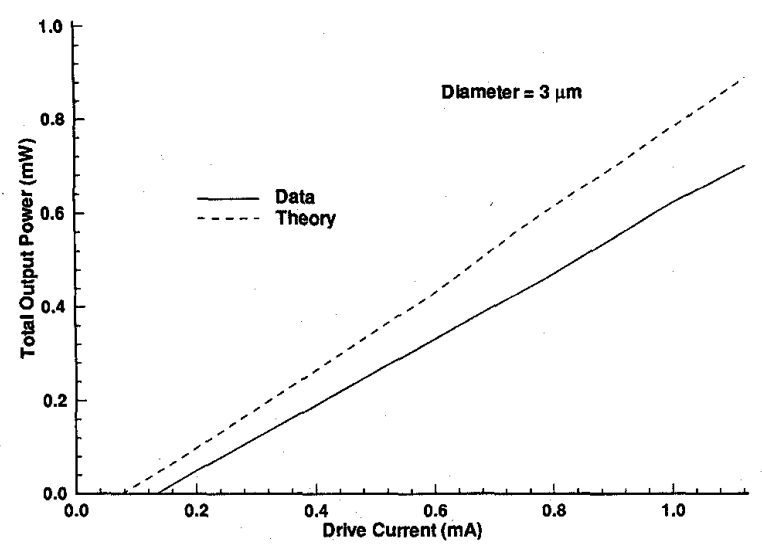

Fig. 9. Measured and predicted LI curves for a $3-\mu \mathrm{m}$-diameter index-guided device of the type shown in Fig. 7. The measured lower efficiency and higher threshold (as compared with the theoretical curve) are believed to result from scattering from the low-index oxide layer not accounted for in the model.

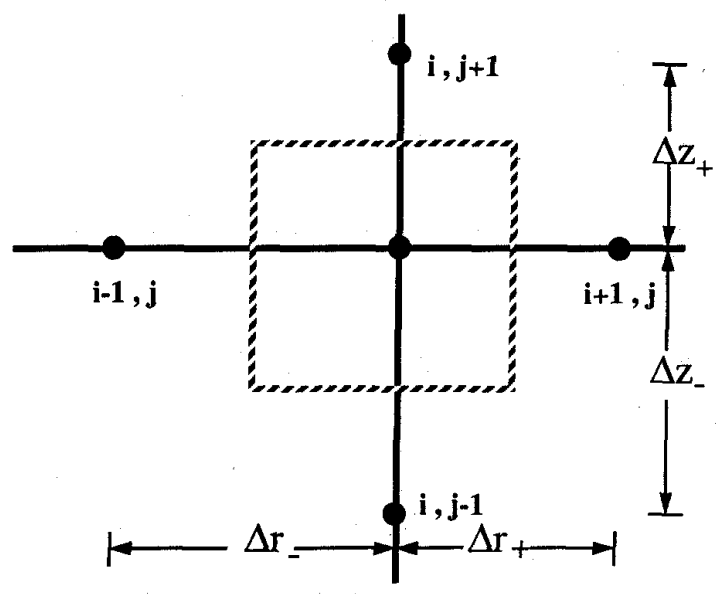

Fig. 10. Finite difference mesh notation and region of integration for the transport (A1).

\section{CONCLUSION}

In summary, we have presented a comprehensive model for the simulation of vertical-cavity surface-emitting semiconductor lasers that calculates the transport of both heat and carriers as well as the behavior of the multilateral-mode optical fields and their interaction with the quantum well gain region. The resulting model has successfully predicted the threshold currents, output powers, and lateral mode behavior of gainguided VCSEL's, including thermal rollover effects at high injection currents. Considerable success has also been achieved at predicting and understanding the impact of thermal lensing on the onset of higher-order lateral modes. For the strongly index-guided devices fabricated with selective oxidation, the model still predicts thresholds and efficiencies well for the large diameter $(7 \mu \mathrm{m})$ case. However, index guiding in these devices results from a single low-index layer that causes both scattering and confinement. Because this layer strongly perturbs the shape of the field profile, the separability assumption used in the derivation of the effective index equations is violated, and the method does not predict the scattering, leading to inaccuracies in predicted device behavior that are the most noticeable for the small diameter $(3 \mu \mathrm{m})$ lasers. Accurate predictions of the model behavior of these devices may require a more sophisticated optical model.

\section{APPENDIX}

Here we derive the finite difference equations used to solve (1). We begin by integrating the latter equation over the dashed region shown in Fig. 10. Remembering the cylindrical symmetry, the formal result is

$$
\oint \bar{\kappa} \nabla \psi \cdot d A=\int S d V
$$

where the area and volume integrals refer to the volume of revolution produced by revolving the dashed area through an angle of $2 \pi$. The resulting difference equation is thus

$$
\begin{aligned}
\pi r_{i-(1 / 2)} & \left(\Delta z_{j}+\Delta z_{j-1}\right) \kappa_{r, i-(1 / 2), j} \frac{\left(\psi_{i, j}-\psi_{i-1, j}\right)}{\Delta r_{i-1}} \\
- & \pi r_{i+(1 / 2)}\left(\Delta z_{j}+\Delta z_{j-1}\right) \kappa_{r, i+(1 / 2), j} \\
& \cdot \frac{\left(\psi_{i+1, j}-\psi_{i, j}\right)}{\Delta r_{i}}+\pi\left(r_{i+(1 / 2)}^{2}-r_{i-(1 / 2)}^{2}\right) \\
& \cdot\left[\kappa_{z, i, j-(1 / 2)} \frac{\left(\psi_{i, j}-\psi_{i, j-1}\right)}{\Delta z_{j-1}}-\kappa_{z, i, j+(1 / 2)}\right. \\
& \left.\cdot \frac{\left(\psi_{i, j+1}-\psi_{i, j}\right)}{\Delta z_{j}}\right] \\
= & -\pi\left(r_{i+(1 / 2)}^{2}-r_{i-(1 / 2)}^{2}\right) \times \frac{\left(\Delta z_{j}+\Delta z_{j-1}\right)}{2} S_{i, j},
\end{aligned}
$$

where for simplicity we have assumed the transport tensor to have only two diagonal elements, $\kappa_{r}$ and $\kappa_{z}$. Equation (A2) is solved using the iterative two-step ADI algorithm [27] that may be described symbolically by the equations

$$
\begin{aligned}
& \left(\omega_{n}+\frac{\partial}{\partial z} \kappa_{z} \frac{\partial}{\partial z}\right) \psi^{n+(1 / 2)} \\
& =\left(\omega_{n}-\frac{1}{r} \frac{\partial}{\partial r}\left(r \kappa_{r} \frac{\partial}{\partial r}\right)\right) \psi^{n}-S \\
& \left(\omega_{n}+\frac{1}{r} \frac{\partial}{\partial r}\left(r \kappa_{r} \frac{\partial}{\partial r}\right)\right) \psi^{n+1} \\
& =\left(\omega_{n}-\frac{\partial}{\partial z} \kappa_{z} \frac{\partial}{\partial z}\right) \psi^{n+(1 / 2)}-S
\end{aligned}
$$

with the differential operators replaced by their finite difference counterparts from (A2). In (A3) and (A4), the superscripts refer to iteration level, and $\omega_{n}$ is a two-level sequence of acceleration parameters [27]. These parameters are approximately given by

$$
\begin{aligned}
& \omega_{1}=-\sqrt{2 R} \frac{\sqrt{1+R^{2}}+R-1}{L^{2}} \\
& \omega_{2}=-\sqrt{2 R} \frac{\sqrt{1+R^{2}}-R+1}{L^{2}}
\end{aligned}
$$

where $L$ is the maximum problem extent, and $R$ the ratio of the latter to the minimum mesh size. However, (A5) should be interpreted only as a guide, and may not represent optimum 
convergence rates. The latter are best determined by trial and error for a specific problem. Alternatively, (A2) may be solved by direct inversion of the band matrix using commerciallyavailable matrix inversion routines. This latter approach has been found to be generally faster unless an accurate initial guess for the solution is available. However, more storage is required for direct inversion, with a $75 \times 100$ mesh requiring 10 Mbytes of memory (currently within the range of most PC's).

\section{REFERENCES}

[1] D. B. Young, J. W. Scott, F. H. Peters, M. G. Peters, M. L. Majewski, B. J. Thibeault, S. W. Corzine, and L. A. Coldren, "Enhanced performance of offset-gain high-barrier vertical-cavity surface-emitting lasers," IEEE J. Quantum Electron., vol. 29, no. 6, pp. 2013-2022, 1993.

[2] K. L. Lear, S. P. Kilcoyne, and S. A. Chalmers, "High power conversion efficiencies and scaling issues for multimode vertical-cavity top-surfaceemitting lasers," IEEE Photon. Technol. Lett., vol. 6, no. 7, pp. 778-781, 1994.

[3] K. L. Lear, K. D. Choquette, R. P. Schneider, Jr., S. P. Kilcoyne, and K. M. Geib, "Selectively oxidized vertical cavity surface emitting lasers with $50 \%$ power conversion efficiency," Electron. Lett., vol. 31, no. 3, pp. 208-209, 1995.

[4] J. A. Lott, R. P. Schneider, Jr., K. D. Choquette, S. P. Kilcoyne and J. J. Figiel, "Room temperature continuous wave operation of red vertical cavity surface emitting laser diodes," Electron. Lett., vol. 29, pp. $1693-1694,1993$

[5] R. P. Schneider, Jr., K. D. Choquette, J. A. Lott, K. L. Lear, J. J. Figiel, and K. J. Malloy, "Efficient room-temperature continuous-wave AIGaInP/AlGaAs visible $(670 \mathrm{~nm})$ vertical-cavity surface-emitting laser diodes," IEEE Photon. Technol. Lett., vol. 6, no. 3, pp. 313-316, 1994.

[6] J. W. Scott, S. W. Corzine, D. B. Young, and L. A. Coldren, "Modeling the current-to-light characteristics of index-guided vertical-cavity surface-emitting lasers," App. Phys. Lett, vol. 62, no. 10, pp. 1050-1052, 1993.

[7] J. W. Scott, R. S. Geels, S. W. Corzine, and L. A. Coldren, "Modeling temperature effects and spatial hole burning to optimize vertical-cavity surface-emitting laser performance," IEEE J. Quantum Electron., vol. 29, no. 5, pp. 1295-1308, 1993

[8] W. W. Chow, R. P. Schneider, Jr,, J. A. Lott, and K. D. Choquette, "Wavelength dependence of the threshold in an InGaP.InAlGaP vertical cavity surface emitting laser," Appl. Phys. Lett., vol. 65, no. 2, pp. $135-137,1994$.

[9] D. I. Babic, R. J. Ram, J. E. Bowers, M. Tan, and L. Yang, "Scaling laws for gain-guided vertical cavity lasers with distributed Bragg reflectors," Appl. Phys. Lett., vol. 64, no. 14, p. 1762-1764, 1994.

[10] J. Piprek, H. Wenzel, and G. Sztefka, "Modeling thermal effects on the light vs. current characteristic of gain-guided vertical-cavity surfaceemitting lasers," IEEE Photon. Technol. Lett., vol. 6, no. 2, pp. 139-142, 1994.

[11] J.-P. Zhang and K. Petermann, "Beam propagation model for verticalcavity surface-emitting lasers: threshold properties," IEEE J. Quantum Electron., vol. 30, no. 7, pp. 1529-1536, 1994.

[12] M. Shimizu, F. Koyama, and K. Iga, "Transverse mode analysis for surface emitting laser using beam propagation method," IEICE Trans., vol. E 74, no. 10, pp. 3334-3341, 1991.

[13] R. Michalzik and K. J. Ebeling, "Modeling and design of protonimplanted ultralow-threshold vertical-cavity laser diodes," IEEE $J$. Quantum Electron., vol. 29, no. 6, pp. 1963-1973, 1993.

[14] L. E. Thode, G. Csanak, L. L. So, and T. J. T. Kwan, "Time-dependent numerical simulation of vertical cavity lasers," in Proc. SPIE, Symp. Physics and Simulation of Optoelectron. Devices II, Los Angeles, CA, Jan. 24, 1994, vol. 2146, pp. 174-184.

[15] G. R. Hadley, J. P. Hohimer, and A. Owyoung, "Comprehensive modeling of diode arrays and broad-area devices with applications to lateral index tailoring," IEEE J. Quantum Electron., vol. 24, no. 11, pp. $2138-2152,1988$.

[16] W. H. Press, S. A. Teukolsky, W. T. Vetterling, and B. P. Flannery, Numerical Recipes in FORTRAN, 2nd ed. New York: Cambridge University Press, p. 861.

[17] A. E. Siegman, Lasers. Mill Valley, CA: University Science Books, p. 430 .

[18] S. W. Corzine, R. H. Yan, and L. A. Coldren, "Optical gain in III-V bulk and quantum well semiconductors," in Quantum Well Lasers, P. S. Zory, Ed. New York: Academic, 1993, ch. 1.

[19] $\_$, "Theoretical gain in strained InGaAs/AlGaAs quantum wells including valence-band mixing effects," Appl. Phys. Lett., vol. 57, p. $2835,1990$.

[20] J. M. Luttinger and W. Kohn, "Motion of electrons and holes in perturbed fields," Phys. Rev., vol. 97, p. 869, 1955.

[21] D. A. Broido and L. J. Sham, "Effective masses of holes at GaAsAlGaAs heterojunctions," Phys. Rev., vol. B 31, p. 888, 1985.

[22] D. Ahn and S. L. Chuang, "Optical gain in a strained-layer quantum-well laser," IEEE J. Quantum Electron,, vol. 24, p. 2400, 1988.

[23] S. R. Chinn, P. S. Zory, and A. R. Reisinger, "A model for GRINSCH-SQW diode lasers," IEEE J. Quantum Electron., vol. 24, p. 2191, 1988.

[24] S. Y. Hu, D. B. Young, S. W. Corzine, A. C. Gossard, and L. A. Coldren, "High efficiency and low threshold InGaAs/AlGaAs quantum well lasers," J. Appl. Phys., vol. 76, p. 3932, 1994.

[25] P. M. Norris, G. Chen, and C.-L. Tien, "Size effects on the temperature rise in vertical-cavity surface-emitting laser diodes," Int. J. Heat Mass Transfer, vol. 37, suppl. 1, pp. 9-17, 1994.

[26] K. L. Lear, K. D. Choquette, R. P. Schneider, Jr., and S. P. Kilcoyne, "Modal analysis of a small surface-emitting laser with a selectively oxidized waveguide," Appl. Phys. Lett., vol. 66, p. 2616, 1995.

[27] R. S. Varga, Matrix Iterative Analysis. Englewood Cliffs, NJ: PrenticeHall, 1963, ch. 7, p. 212.

G. Ronald Hadley (SM'93), photograph and biography not available at the time of publication.

K. L. Lear (S'83-M'88), photograph and biográphy not available at the time of publication. -

M. E. Warren, photograph and biography not available at the time of publication.

K. D. Choquette, photograph and biography not available at the time of publication.

J. W. Scott, photograph and biography not available at the time of publication.

S. W. Corzine, photograph and biography not available at the time of publication. 